\title{
Control Strategy of Dual-Drive Powertrain System of Pure Electric Vehicle based on Real-Time Optimization
}

\author{
Yong Wang ${ }^{*}$ and Jiabin Deng \\ Department of Intelligent Manufacturing and Automotive, Chongqing College of Electronic Engineering, Chongqing, 401331, China
}

\begin{abstract}
Dual-driving powertrain system of pure electric vehicle has two power sources and the main function of power control strategy for finished vehicle is: power distribution in order to control operating condition of all components for the powertrain system according to operating characteristics of powertrain system and components of finished vehicle as well as driving status data of vehicles. In overall consideration of the impacts of efficiency of all components for powertrain system and system efficiency in different operating patterns on efficiency of finished vehicle, control strategy of pattern division and power distribution is based on optimum efficiency of driving system in order to guarantee basic performance of finished vehicle and increase driving range of finished vehicle. MATLAB/Simulink simulation analysis software is used to establish simulation platform for powertrain system of pure electric vehicle with two driving force and to carry out simulation verification for control strategy of finished vehicle. The results show that all power components can be put into synergetic operation and vehicles will have higher performance, energy saving effects as well as efficient and reasonable control with the control strategy which has been developed.
\end{abstract}

Keywords: electric vehicle; control strategy; real-time optimization; Dual-driving powertrain system

(Submitted on May 22, 2019; Revised on October 10, 2019; Accepted on November 24, 2019)

(C) 2019 Totem Publisher, Inc. All rights reserved.

\section{Introduction}

Powertrain system of pure electric vehicle with two driving force is a new-type drive system of electric automobile and the power coupling mechanism consists of single -planetary gear mechanism, clutch and brake etc. Brake, clutch and two power sources can be controlled to realize several operating patterns: coupling operating pattern of revolving speed of two motors and torque and single operation pattern of single motor. These patterns can be adopted to the expand scope of working area with high drive efficiency of power source to great extent and realize ECVT function at the same time. In coupling drive of double motors through revolving speed, stepless speed change of powertrain system can be realized to break through restrictions of gear of step speed change on operating area of power source. Besides, speed ratio variation range of speed control system can be expanded to increase driving range; if driving safety can be guaranteed, breaking energy recovery efficiency of automobile can be increased to further increase driving range of pure electric vehicle.

As for electric vehicle with multiple power sources, energy management strategy is to set a high-level control algorithm in order to determine the power suitable for production and the power for distribution of different power sources. It can be also called power control strategy and plays a very important role in improving economic efficiency and dynamic property of energy consumption [1]. Powertrain system of pure electric vehicle with two driving force has two power sources and the main function of power control strategy for finished vehicle is: power distribution in order to control operating condition of all components (drive motor, clutch/brake and so on) for the powertrain system according to operating characteristics of powertrain system and components of finished vehicle as well as driving status data of vehicles. In overall consideration of the impacts of = efficiency of all components for powertrain system and system efficiency in different operating patterns on efficiency of finished vehicle, research on control strategy of pattern division and power distribution based on optimum efficiency of driving system is of great significance to guarantee basic performance of finished vehicle and increase driving range of finished vehicle.

\footnotetext{
* Corresponding author.

E-mail address: wycowboy@163.com
} 
Powertrain system of pure electric vehicle with single motor has only one power source and the research on control strategy is mainly torque analysis according to drivers' demands, namely, how to execute drivers' command according to powertrain system. Wang Jia puts forward one control structure with multiple inputs and single output to set pure electric vehicle into three operating patterns and fuzzy control method is proposed to determine percentage of output torque. The results show that the control strategy is a good way to increase overall performance of pure electric vehicle [2]. When Zhang Qing sets a control strategy for pure electric vehicle, fuzzy recognizer is used for pattern recognition of drivers' driving intention and the torque optimization and control strategy in three driving patterns is set through linear computation, torque compensation and power assembly efficiency optimization. The results show that the optimization and control strategy will greatly increase dynamic property and economic efficiency of finished vehicle [3]. Chen Yong proposes that optimization can be realized through self-adaptation simulated annealing and generalized simple gradient algorithm with multi-disciplinary design optimization idea, with energy consumption optimization of pure electric vehicle as the objective and dynamic property and driving range as constraint conditions. The results indicate that the optimization method can be adopted to realize design objective [4]. Based on pattern of dual-motor coupling driving system for pure electric bus, Shuo Zhang adopts dynamic gauge-oriented algorithm for optimal design of control strategy for finished vehicle and thus rules of pattern of dual-motor coupling driving system have been determined. It is proved that the control strategy prepared according to the method is efficient and feasible [5]. According to the proposed dual-motor coupling driving system, Han Shengming converts drivers' operating intention into required torque of dual motors and genetic algorithm is adopted to optimize control strategy of mode switch, which shows the control strategy is efficient and feasible [6]. Coronado PDU has put forward rule-based control strategy according to dual-motor driving system of pure electric vehicle and then calculated and compared energy consumption of finished vehicle in each operating pattern in order to prepare the optimal operating pattern switch strategy [7]. Qin Datong puts forward control strategy of driving torque compensation for pure electric vehicle and the control strategy is based on determination of basic driving torque. Fuzzy algorithm is adopted to recognize drivers' intention and accelerated compensation torque is set according to recognition results. Results show that the control strategy can effectively promote dynamic property of finished vehicle [8]. In terms of control through multiple power sources for electric automobile with hybrid power, the research is mainly about global optimum control strategy, instant optimum control strategy and rule-based control strategy [9-10]. Powertrain system of pure electric vehicle with two driving force has two power sources and we can use control thought of electric automobile with hybrid power for reference.

\section{Structure and Operating Principles of Dual-Driving Powertrain System}

In this paper, one new-pattern transmission system of pure electric vehicle with two driving force is designed according to dynamic coupling function of planetary gear mechanism and clutch, brake and other joint elements are used to realize impeccable operating pattern and flexible switchover between operating patterns. The overall structure and principle of new-pattern transmission system of pure electric vehicle with two driving force is shown in Figure 1.

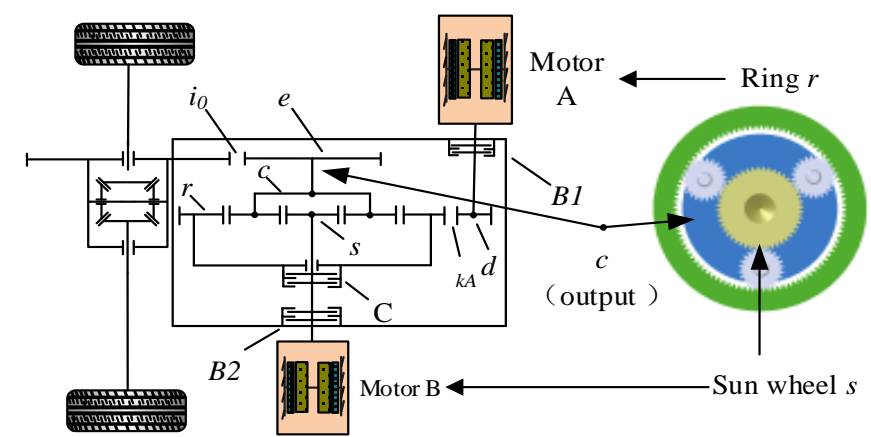

Figure 1. The diagrammatic sketch of dual drive powertrain for pure electric vehicle

The system consists of one single-row planetary gear mechanism, two motors (motor A and motor B), two wet brakes (B1 and B2) and one wet clutch C and the power output is completed through planetary gear coupling devices. Sun wheel s of single-row planetary gear mechanism is directly connected with output shaft of motor B; gear ring $\mathrm{r}$ is connected with output shaft of motor A through transmission gear d; planetary carrier c is connected with drive axle through gear e for the purpose of power output; gear ring $\mathrm{r}$ can be connected with sun wheel s through clutch $\mathrm{C}$; the movable part of brake B1 can be connected with box and the fixed part can be connected with output shaft of motor A and brake B1 can be used to brake ring c; the movable part of brake B2 can be connected with box and the fixed part can be connected with output shaft of motor B and then brake B1 can be used to brake sun wheel s.

Switchover of several operating patterns can be realized through braking/separation of sun wheel and gear ring as well 
as connection/separation of clutch. The following nine operating patterns are set according to operating conditions of motor A, motor B, brake and clutch, as shown in Table 1.

Table 1 . The mode summary of dual drive powertrain for pure electric vehicle

\begin{tabular}{|c|c|c|c|c|c|c|}
\hline Work mode & Motor A & Motor B & B1 & B2 & $\mathrm{C}$ & Vehicle state \\
\hline Parking braking/neutral position & 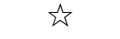 & $\hat{z}$ & $\circ$ & $\circ$ & ० & $\mathrm{P} / \mathrm{N}$ \\
\hline Motor A driving & $\star$ & $\hat{s}$ & $\circ$ & $\bullet$ & ० & Start, medium and low speed \\
\hline Motor B driving & $\sum$ & $\star$ & $\bullet$ & $\circ$ & $\circ$ & Low speed and heavy load \\
\hline Speed coupled driving & $\star$ & $\star$ & ० & $\circ$ & ० & $\begin{array}{l}\text { Medium and high speed, medium } \\
\text { load }\end{array}$ \\
\hline Motor A braking & $\star$ & $\hat{z}$ & $\circ$ & $\bullet$ & $\circ$ & Braking and taxiing \\
\hline Motor B braking & 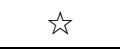 & $\star$ & $\bullet$ & $\circ$ & ० & Braking and taxiing \\
\hline Speed coupled braking & $\star$ & $\star$ & ० & $\circ$ & ० & Braking and taxiing \\
\hline Torque coupled braking & $\star$ & $\star$ & $\circ$ & $\circ$ & $\bullet$ & Braking and taxiing \\
\hline
\end{tabular}

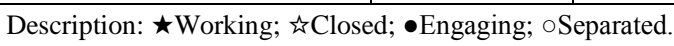

\section{Analysis on Drivers' Required Torque}

As for traditional vehicles, after the gear is determined, drivers will control the vehicle through accelerator pedal and the aperture change of accelerator pedal reflects drivers' expectation of vehicles' driving status, namely expectation of vehicle power, which is the same as pure electric vehicle. It is necessary to convert drivers' pedal operation into specific torque command of power source, namely analysis strategy of drivers' required torque. At present, torque demands of pure electric vehicle drivers are generally determined according to external characteristics of power source and load coefficient of required torque. Load factor of required torque is the function of accelerator pedal aperture and it mainly includes three kinds as shown in Figure 2. Curve 1 is linearity curve which reflects linear relation between load factor of required torque and accelerator pedal aperture. This relation can meet basic driving demands but driving operation is so-so; Curve 2 is the convex curve and the relation between load factor of required torque and accelerator pedal is obvious than that in Curve 1 and this relation is "relatively fixed". Torque regulation is more sensitive in order to highlight characteristics of dynamic property; Curve 3 is the downward protrusion curve in which the relation between load factor of required torque and accelerator pedal is not as obvious as that in Curve 1 and this relation is "relatively flexible". The torque regulation is not so sensitive in order to highlight characteristics of economic efficiency.

Load factor of drivers' required torque is not only related to aperture of accelerator pedal but also related to vehicle speed. At low speed, this relation is "relatively fixed" in order to increase sensitivity; at high speed, this relation is "relatively flexible" in order to reduce sensitivity. To solve the problem of considering conventional required torque and analysis of all properties of vehicle, characteristics of above-mentioned three torque analysis strategies are taken into consideration in this paper and load factor model of economical and mobile torque is proposed according to project experience. Moreover, two analysis models of drivers' required torque have been established according to operating characteristics of drive motor system at different aperture of accelerator pedal and different vehicle speeds. Drivers will choose one through two keys of economical and mobile patterns, as shown in Figure 3. It can be seen from the figure that the slope of relation between accelerator pedal and required torque in mobile curve is greater than that in economical curve; at high aperture of accelerator pedal, required torque tends to be the maximum value; at low aperture of accelerator pedal, the torque difference between two adjacent pedals is great, which reflects rapid response of torque.

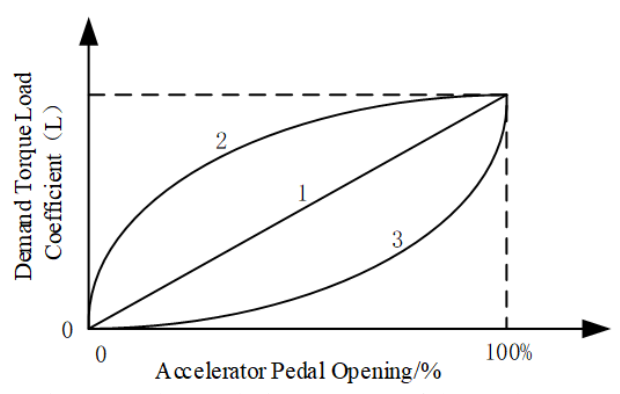

Figure 2. The resolution strategy of demand torque 


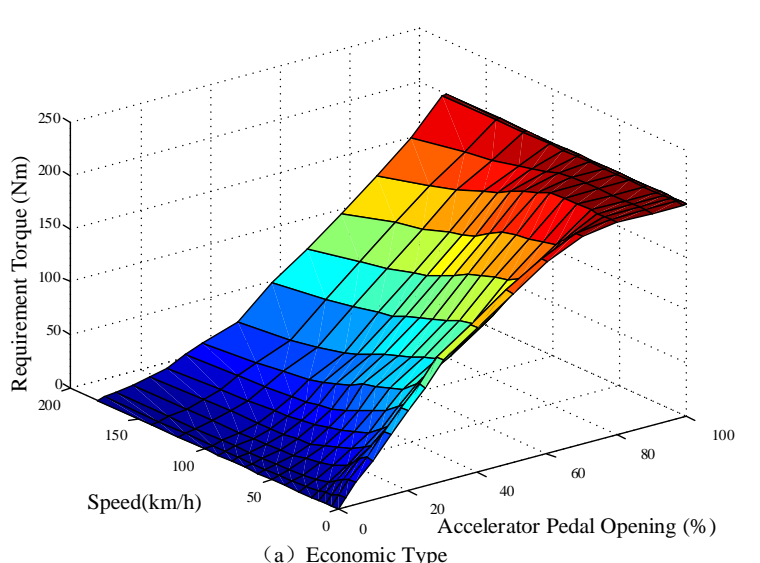

Figure 3. The demand torque model

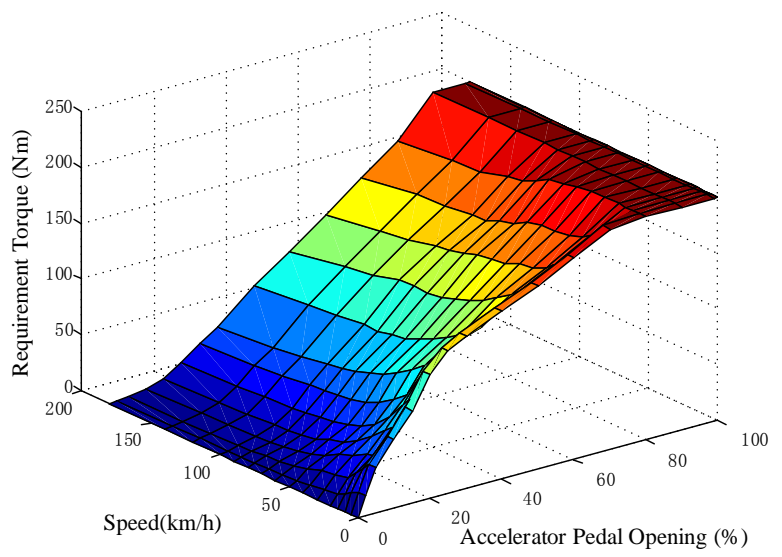

(b) Power Type

\section{Control Strategy of Optimal Real-Time Efficiency based on Logic Threshold}

After analysis strategy of drivers' required torque is determined, we need to determine its operating pattern and power distribution scheme. Control strategy of optimal real-time efficiency based on logic threshold is: logic threshold will be determined and area under operating pattern will be divided according to analysis on driving system efficiency. Within working area of torque coupling and revolving speed coupling, system efficiency will be determined for real-time optimization in order to determine optimum efficiency of driving system, operating points of two motors and to realize optimum control of efficiency.

\subsection{Efficiency Analysis of Dual-Driving Powertrain System}

Powertrain system of pure electric vehicle with two driving force is different from other pure electric vehicles and it has several operating patterns. In operation under single-motor pattern, the operating point will be determined according to speed of finished vehicle and required torque and the efficiency of driving system will be accordingly determined; in operation under dual-motor torque coupling pattern, the revolving speed of two motors will be proportional and determined according to speed of finished vehicle and parameter of transmission system. However, the torque can be arbitrarily combined within allowable range; in operation under coupling pattern of revolving speed, the torque of two motors will be proportionally distributed and determined according to the required torque. However, the revolving speed can be arbitrarily combined within allowable range.

Efficiency of driving system for pure electric vehicle is related to efficiency of drive motor, controller, power battery and transmission system. Driving force of finished vehicle is shown in Equation (1):

$$
F_{\mathrm{r}}=m g f_{\mathrm{r}} \cos \alpha+m g \sin \alpha+\frac{C_{\mathrm{D}} A u^{2}}{21.15}+\delta m \frac{\mathrm{d} u}{\mathrm{~d} t}
$$

In which, $\alpha$ - Road grade, rad.

Overall efficiency of driving system is shown in Equation (2):

$$
\eta_{\mathrm{sys}}=\frac{P_{\text {out }}}{P_{\text {in }}}=\frac{F_{\mathrm{r}} \cdot u / 1000}{\left(T_{\mathrm{m} 1} \omega_{\mathrm{m} 1}+T_{\mathrm{m} 2} \omega_{\mathrm{m} 2}\right) \cdot \eta_{\mathrm{m}} \cdot \eta_{\mathrm{b}} \cdot \eta_{\mathrm{T}}}
$$

In which, $\eta_{\mathrm{sys}}$ - Overall efficiency of driving system.

\subsection{Efficiency of Driving System in Single-Motor Operating Pattern}

In single-motor operating pattern, the revolving speed of motor can be determined according to vehicle speed and parameters of transmission system and the revolving speed can be determined according to required torque of finished 
vehicle. In this case, efficiency of motor system and power battery system can be checked from MAP diagram of motor efficiency and power battery efficiency. In this pattern, the overall efficiency of driving system is shown in Equation (3):

$$
\eta_{\mathrm{sys}}=\frac{P_{\text {out }}}{P_{\text {in }}}=\frac{F_{\mathrm{r}} \cdot u / 1000}{T_{\mathrm{m}} \cdot \omega_{\mathrm{m}} \cdot \eta_{\mathrm{m}} \cdot \eta_{\mathrm{b}} \cdot \eta_{\mathrm{T}}}
$$

In which, $T_{\mathrm{m}}$ - Operating torque of motor, $\mathrm{Nm}, \omega_{\mathrm{m}}$ - Speed of motor, $\mathrm{r} / \mathrm{min}$.

\subsection{Efficiency of Driving System in Dual-Motor Coupling Operating Pattern}

As mentioned before, if operating points of two motors are not determined when two motors are in coupling drive, it is necessary to adopt optimization algorithm to determine optimum distribution method of two motors and to determine operating points of motors. Optimized objective function of efficiency is shown in Equation (4):

$$
f(x)=\max \eta_{\text {sys }}
$$

Constraint conditions are shown in Equation (5):

$$
\left\{\begin{array}{l}
0 \leq\left|P_{\mathrm{b}}\right| \leq P_{\mathrm{b} \max } \\
S O C_{\min } \leq S O C \\
U_{\mathrm{bmin}} \leq U_{\mathrm{bat}} \leq U_{\mathrm{bmax}} \\
0 \leq\left|T_{A}\left(\omega_{A}\right)\right| \leq T_{\max A}\left(\omega_{A}\right) \\
0 \leq\left|T_{B}\left(\omega_{B}\right)\right| \leq T_{\max A}\left(\omega_{B}\right) \\
0 \leq \omega_{A} \leq \omega_{\max A} \\
0 \leq \omega_{B} \leq \omega_{\max B}
\end{array}\right.
$$

In which, $S O C_{\min }$ - Minimum $S O C$ of battery pack, $U_{\text {bmin }}, U_{\text {bmax }}$ - Maximum voltage and minimum voltage of battery pack $\mathrm{V}$.

\subsection{Regional Division in Operating Pattern}

Based on above-mentioned analysis of driving efficiency, the surface diagram will be prepared according to information about system efficiency and operating points of motor in single operating pattern of single motor, torque coupling operating pattern of dual motors and coupling operating pattern of revolving speed, as shown in Figure 4(a). This diagram will be projected to the plane of vehicle speed and required torque to determine working area of optimum efficiency of Dualdriving powertrain system. Figure 4(b) illustrates regional division strategy of operating pattern in driving conditions.

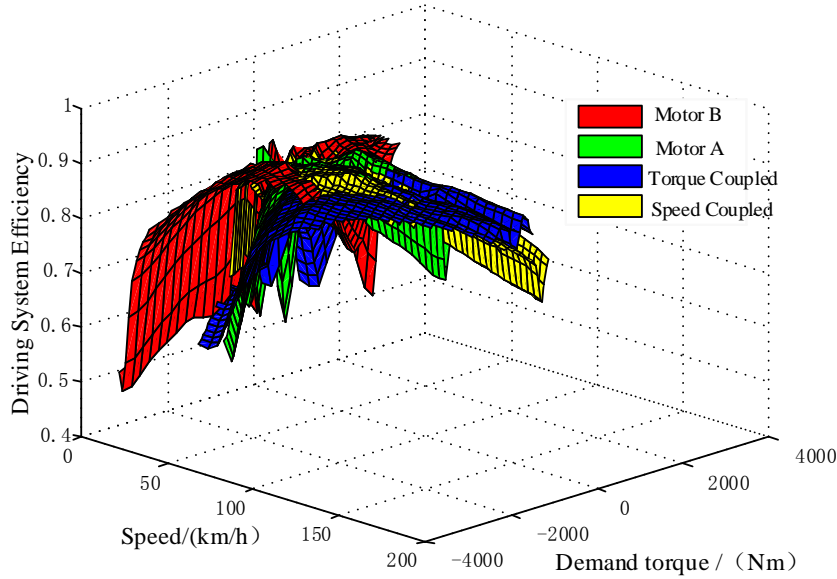

(a)

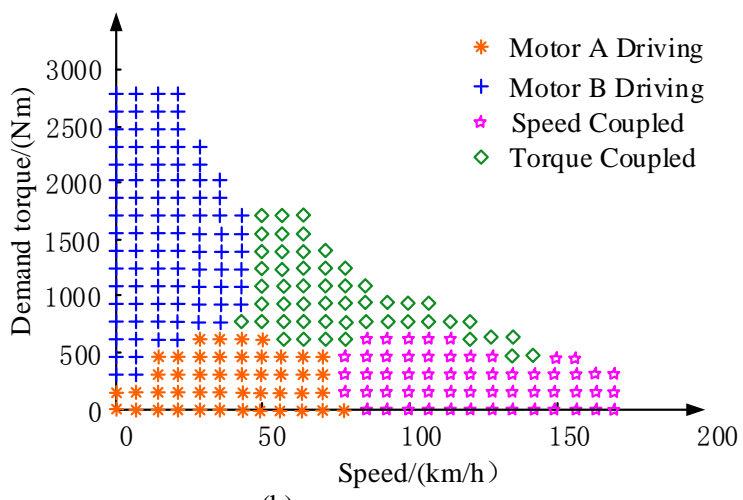

(b)

Figure 4. The system efficiency surface and model strategy 
It can be seen from the figure that in single operating pattern, motor $\mathrm{A}$ is in operation at medium and low speed as well as low demand of torque; in single operating pattern, motor B is in operation at medium and high speed as well as high demand of torque; in torque coupling operating pattern of dual motors, the motor is mainly in operation at medium and high speed as well as high demand of torque. Motor B makes up deficiency of insufficient power of motor A at high speed; in coupling operating pattern of revolving speed, the motor is mainly in operation at medium and high speed as well as low demand of torque.

\subsection{Power Distribution of Dual Motors}

According to analysis in Section 4.4.2, regional division scheme in operating pattern can be adopted to ensure optimum efficiency of transmission system of pure electric vehicle with two driving force prepared to determine operating points of two motors in each operating pattern, namely objective torque and speed of motors. If transmission system of pure electric vehicle with two driving force is in single operating pattern of single motor, motor torque and speed will be respectively determined according to drivers' required torque and speed of finished vehicle; in operating pattern under coupling drive of dual motors, operating points of two motors are not fixed and optimum distribution will be carried out according to realtime system efficiency. Optimum power distribution scheme can be determined through optimal computation in operating pattern of torque coupling, as shown in Figure 5(a). The optimum distribution scheme of revolving speed in coupling operating pattern of revolving speed is shown in Figure 5(b).
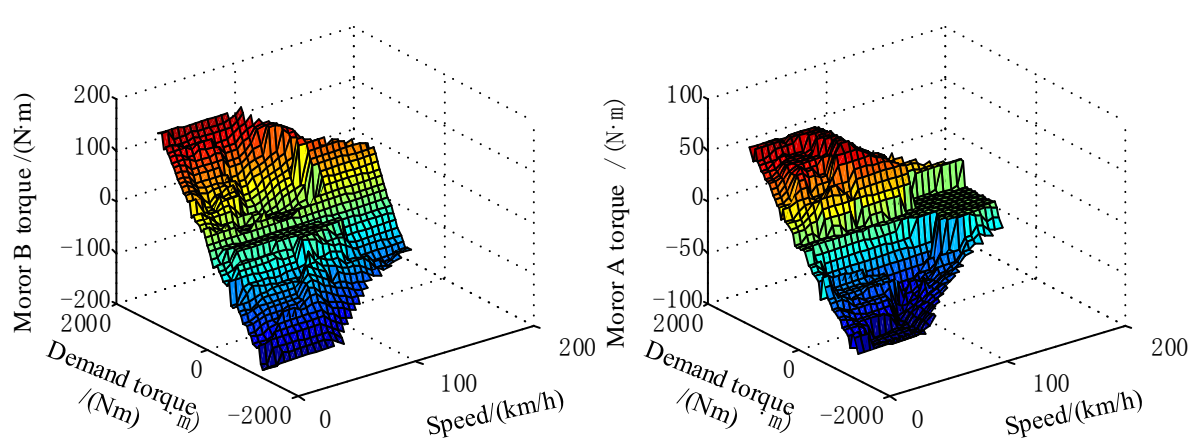

(a)
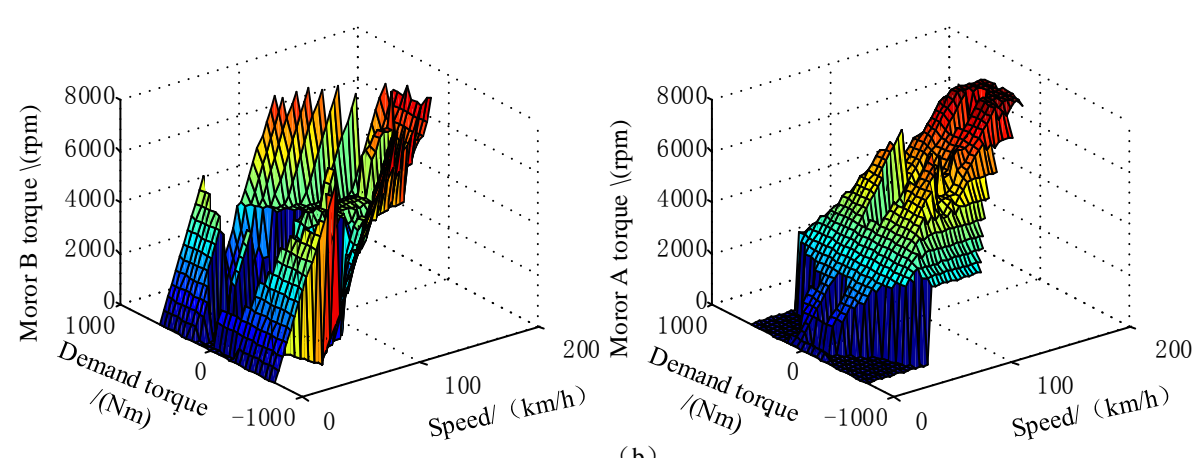

(b)

Figure 5. Optimal power distribution strategy for torque and speed coupling

\section{Simulation of Control Strategy and Result Analysis}

To verify reasonability of above-mentioned control strategy, the simulation model for transmission system of pure electric vehicle with two driving force is established on MATLAB/Simulink platform for simulation analysis of control strategy for cyclic operation. It is worth noting that pattern switch process is namely control strategy during lower pattern switch and it belongs to transient problem. A lot of computing resources will be consumed during computation of dynamic property and economical efficiency and its influences on dynamic property and economic efficiency are limited, which mainly influences driving smoothness and driving sense. Therefore, these influences can be ignored during simulation in this chapter.

Simulation results based on typical working conditions of NEDC are shown in Figure 6 to Figure 10. Figure 6 reveals working conditions and vehicle speed in cyclic operation of NEDC. Figure 7 and Figure 8 are curve diagram of motor 
torque and revolving speed in cyclic operation of NEDC. Figure 9 is the pattern switch in cyclic operation of NEDC and Figure 10 is about distribution of overall efficiency for Dual-driving powertrain system in cyclic operation of NEDC.

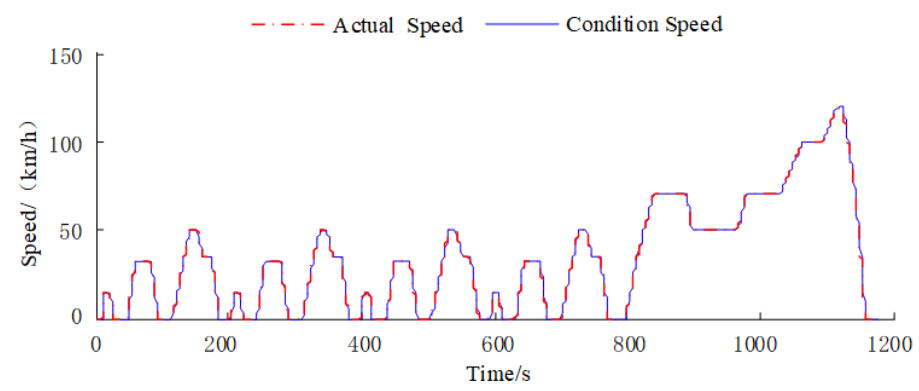

Figure 6. Speed following condition under NEDC cycle condition

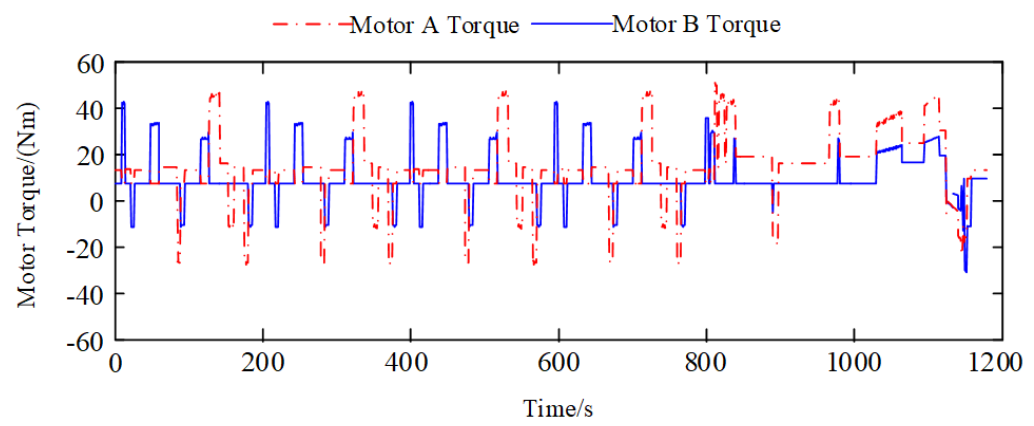

Figure 7. Two motor torque curve under NEDC cycle condition

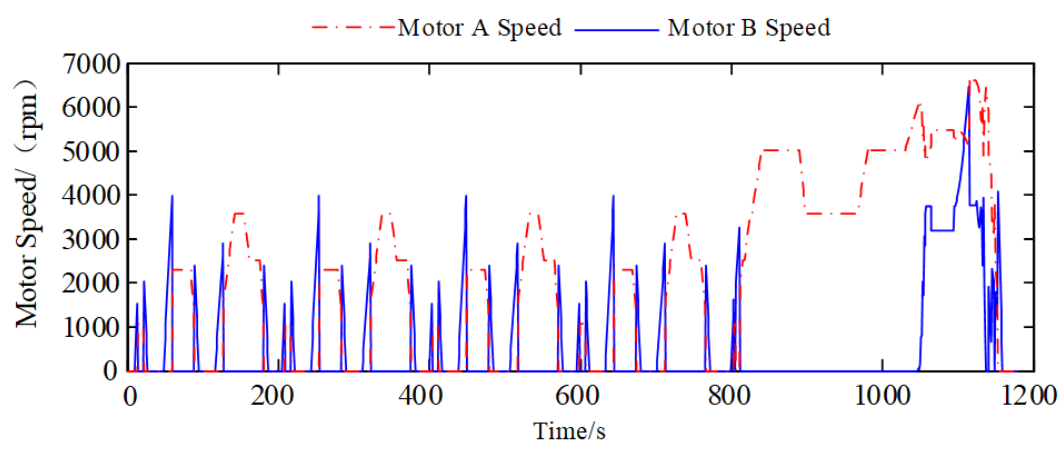

Figure 8. Two motor speed curve under NEDC cycle condition

It can be seen from Figure 6 that the vehicle speed during simulation of transmission system of pure electric vehicle with two driving force can meet vehicle speed demands in cyclic operation, which indicates the driver model is efficient and feasible to meet simulation accuracy demands, simulation model of finished vehicle is in good operation and has higher computing efficiency. It can be seen from Figure 7 and Figure 8 that in cyclic operation, both torque and revolving speed of two motors are in good operation according to established control strategy. At low speed and small load, motor A will be used to complete power output; at low speed and heavy load, motor B will be used to complete power output; at high speed and heavy load, two motors will be put into coupling of revolving speed to complete power output; at medium and high speed as well as heavy load, two motors will be put into coupling of torque to complete power output. Braking and sliding operations are completed in a similar way. Energy will be recovered in the most efficient operating pattern.

In Figure 9, pattern a is the operating pattern when motor $\mathrm{A}$ is in independent drive, pattern $\mathrm{b}$ refers to operating pattern when two motors are in coupling of revolving speed, pattern c refers to operating pattern when two motors are in coupling of torque and pattern d represents operating pattern when motor B is in independent operation. It can be seen from this figure that when the vehicle is started, the system is in pattern a. When torque demands are at upper limit of motor A, the system will be switched to pattern d for continuous acceleration. The first part of NEDC cyclic operation belongs to operating conditions in urban area where vehicle speed is low and in constant changes. Therefore, the main motor A for this system is in independent operating pattern. At great demands of torque, motor A will be switched to motor B for independent operation. At high speed of vehicle, the system will be switched to operating pattern of torque coupling and revolving speed coupling. It also shows that the whole coupling system does not switch patterns frequently, 
which means the delay switch strategy is feasible. It can be seen from Figure 10 that in cyclic operation of NEDC, the whole driving system can be put into operation in area with relatively higher efficiency. According to data statistics, if efficient operating point can be determined when the efficiency is above $85 \%$, efficient operating points at high overall efficiency of Dual-driving powertrain system accounts for about $40 \%$ of all operating points and the average efficiency of system is up to $84.9 \%$.

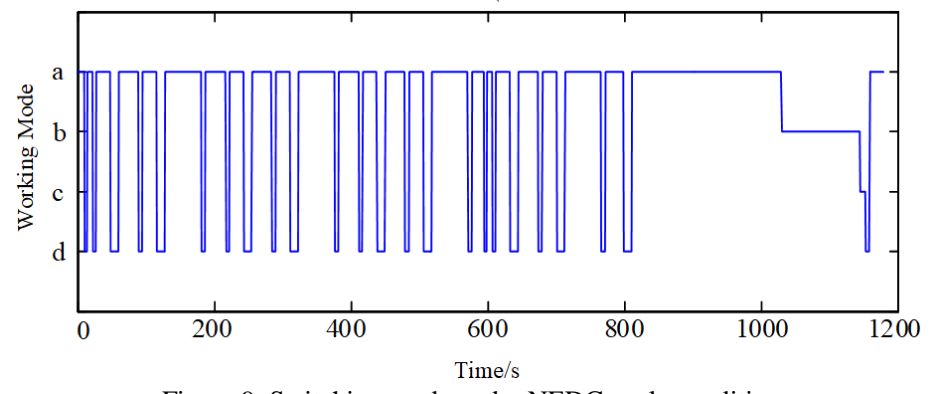

Figure 9. Switching mode under NEDC cycle condition

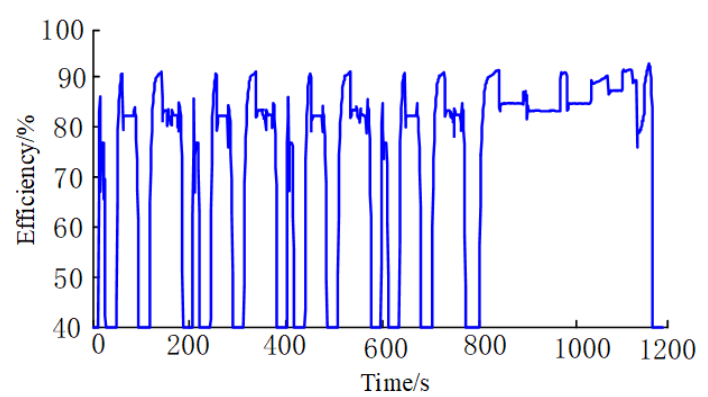

Figure 10. Total efficiency distribution of drive system under NEDC cycle condition

Simulation results show that control process in this paper can be used for efficient analysis of drivers' torque demands and then pattern recognition can be completed according to regional division based on pre-set operating pattern. Next, power will be reasonably distributed. In high efficiency area, transmission system of pure electric vehicle with two driving force can meet driving demands and the finished vehicle has better dynamic property and reasonable driving range. Power performance of finished vehicle totally exceeds design objective. In all cyclic operations, the driving range will increase by nearly $10 \%$ and all power components will be put into synergetic operation with control strategy of optimum real-time efficiency based on logic threshold. With this control strategy, vehicles will be characterized at better performance, obvious energy saving effects as well as efficient and reasonable control.

\section{Conclusions}

In overall consideration of the impacts of efficiency of all components for powertrain system and system efficiency in different operating patterns on efficiency of finished vehicle, control strategy of optimum real-time efficiency is established according to logic threshold and control strategy is proposed for comparative analysis of simulation in order to guarantee basic performance of finished vehicle and increase driving range of finished vehicle. Drivers' required torque will be analyzed to prepare economical and mobile analysis models of drivers' required torque according to existing analysis strategy and project experience; based on principles of instant optimum efficiency of driving system, the working efficiency of Dual-driving powertrain system will be optimized through sequential quadratic programming algorithm to prepare control strategy of optimum real-time efficiency based on logic threshold, including control strategy for regional division of operating pattern and power distribution. The simulation results show that all power components can be put into synergetic operation and vehicles will have higher performance, energy saving effects as well as efficient and reasonable control with the control strategy which has been developed.

\section{Acknowledgements}

The authors would like to express thanks to those who helped in the research work and the preparation of this paper. The authors acknowledge the Scientific and Technological Research Program of Chongqing Municipal Education Commission (Grant: KJQN201803106), Sichuan Provincial Key Lab of Process Equipment and Control (Grant: GK201817), and Chongqing college of electronic engineering (Grant: XJZK201701). 


\section{References}

1. C. C. Lin, H. Peng, J. W. Grizzle, and J. M. Kang, "Power Management Strategy for a Parallel Hybrid Electric Truck," IEEE Transactions on Control Systems Technology, Vol. 32, No. 8, pp. 839-849, June 2003

2. J. Wang, J. Yang, Z. Cai, and X. Zhang, "Optimal Control Strategy of Pure Electric Car based on Fuzzy Control," Automotive Engineering, Vol. 31, No. 4, pp. 362-365, July 2009

3. Q. Zhang, H. Xiong, and L. Yu, "Torque Control Strategy and Simulation of Pure Electric Vehicle," Computer Simulation, Vol. 31, No. 12, pp. 142-146, June 2014

4. Y. Chen, X. Chen, and Y. Lin, "Application of Collaborative Optimization in Multidisciplinary Design Optimization of Electric Vehicles," Automotive Engineering, Vol. 31, No. 8, pp. 709-714, February 2009

5. S. Zhang, C. N. Zhang, G. W. Han, and Q. H. Wang, "Optimal Control Strategy Design based on Dynamic Programming for a Dual-Motor Coupling-Propulsion System," The Scientific World Journal, Vol. 2014, No. 5, February 2014

6. P. D. U. Coronado and H. Ahuett-Garza, "Control Strategy for Power Distribution in Dual Motor Propulsion System for Electric Vehicles," Mathematical Problems in Engineering, Vol. 23, No. 8, pp. 1-10, February 2015

7. D. Qin, M. Zhou, M. Hu, J. Hu, and S. Chen, "Accelerating Torque Compensation Control Strategy for Electric Vehicles," Highway Transportation Science and Technology, Vol. 29, No. 5, pp. 146-151, February 2012

8. X. Ye, Z. Jin, X. Hu, Y. Li, and Q. Lu, "Modeling and Control Strategy Development of a Parallel Hybrid Electric Bus," International Journal of Automotive Technology, Vol. 14, No. 6, pp. 971-985, February 2013

9. Z. Chen and V. Ardalan, "Route Preview in Energy Management of Plug-in Hybrid Vehicles," IEEE Transactions on Control Systems Technology, Vol. 20, No. 2, pp. 546-553, June 2012

10. A. Panday and H. O. Bansal, "A Review of Optimal Energy Management Strategies for Hybrid Electric Vehicle," International Journal of Vehicular Technology, Vol. 23, No. 6, June 2014

Yong Wang received his B.S. degrees in Vehicle engineering from Chongqing University in 2004, his M.S. degree in Vehicle engineering from Chongqing Jiao tong university in 2008 and his Ph.D. degree in Vehicle engineering from Chongqing University in 2018, respectively. Currently, he is an associate professor of Chongqing College of Electronic Engineering. His research interests include automotive automatic transmission system design theory and method of controlling.

Jiabin Deng received his B.S. degrees in Vehicle Engineering of Chongqing University in 2012 and his M.S. degree in Vehicle Engineering of Chongqing University in 2015, respectively. Currently, he is a lecturer and Secondary Technician of Chongqing College of Electronic Engineering. His research interests include: Automobile Maintenance and Automobile Intelligence. 